The Application of Nonlinear Pre-Filters to Prevent Aeroservoelastic Interactions Due to Actuator Rate Limiting

\begin{tabular}{|r|l|}
\hline Journal: & $\begin{array}{l}\text { 53rd AIAA/ASME/ASCE/AHS/ASC Structures, Structural Dynamics, and } \\
\text { Materials Conference }\end{array}$ \\
\hline Manuscript ID: & 1219956 \\
\hline luMeetingID: & 2414 \\
\hline Date Submitted by the Author: & $08-$ Apr-2012 \\
\hline Contact Author: & Alstrom, Bruce; \\
\hline \multicolumn{2}{|l}{} \\
\hline
\end{tabular}

SCHOLARONE"

Manuscripts 


\title{
The Application of Nonlinear Pre-Filters to Prevent Aeroservoelastic Interactions due to Actuator Rate Limiting
}

\author{
Robert Bruce Alstrom ${ }^{1}$, Erik Bollt ${ }^{2}$, Pier Marzocca ${ }^{3}$, Goodarz Ahmadi ${ }^{4}$ \\ Clarkson University, Potsdam, New York 13699-5725
}

\begin{abstract}
In this paper a nonlinear aeroelastic system coupled to a rate limited actuator. The aeroservoelastic system is subjected to a short time sharp edged gust. The piecewise impulse controller was found to provide both state regulation and phase compensation to the rate limited actuator. The closed loop system is largely insensitive to the changes in the phase lead transfer function gain, due to the switching action the controller; but sensitive to the changes in the phase lead transfer function damping ratio. The piecewise impulse controller was found to remove steady state error.
\end{abstract}

\section{INTRODUCTION}

NASA initiated an Integrated Resilient Aircraft Control (IRAC) initiative in 2009 under the Aviation Safety Program [1]. The main thrust of this initiative is to advance the state-of-the-art technology in order to facilitate a design option that allows for increased resiliency to failures, damage, and critical operating conditions. As a particular aspect of this program, adaptive flight control systems have been studied in detail since they will have the capability to automatically adjust the control feedback and command paths to regain stability in the closed loop configuration. One of the consequences of changing the control feedback and command path configuration is the occurrence of Aero-Servo-Elastic (ASE) interaction which results in undesirable Limit Cycle Oscillations (LCO). The combination of changing structural behavior with changing control system gains results in a system with a probability of adverse interactions that is very difficult to predict a priori. Onboard, measurement based methods are required to ensure that the system adjusts to attenuate any adverse ASE interaction before a structural system can become entrained in sustained LCO and vehicle damage occurs. This system must work in concert with the adaptive control system to restore nominal rigid body performance as much as possible without exacerbating the situation with ASE interactions. To that end Li [2] developed an in-flight Narrow Band-Pass Filter (NBWF) detection method that is coupled with an adaptive notch filter that was inserted into the command path to attenuate LCOs in the vehicle flight dynamics. Actuator nonlinearities in general introduce time delays and phase lags between the commanded control surface position and the actual control surface position and as a result induce ASE interactions. Specifically, actuator rate limiting occurs when the input rate to the control surface exceeds the hydraulic and/or mechanical capability of the control surface actuator.

The technological direction is taken here is from research performed on the topic of Pilot Induce Oscillation (PIO). According to MIL-STD-1797A, PIO is defined as the "....sustained or

\footnotetext{
${ }^{1}$ Graduate Student, Department of Mechanical and Aeronautical Engineering, AIAA Senior Member

${ }^{2}$ Professor, Department of Mathematics and Computer Science

${ }^{3}$ Associate Professor, Department of Mechanical and Aeronautical Engineering, AIAA Senior Member

${ }^{4}$ Professor and Dean of School of Engineering, Department of Mechanical and Aeronautical Engineering
} 
uncontrollable oscillations from the efforts of the pilot to control the aircraft" [3]. PIO incidents include the Space Shuttle, YF-22 (now designated the F-22 Raptor) and the JAS-39 Grippen. For the latter two aircraft, rate limiting in the pitch axis resulted in the loss of the latter two air vehicles mentioned previous. Although occurring in a different frequency spectrum, the PIO problem is analogous to the problem of LCOs in primary flight structures. Previous work by Alstrom et al. [4] demonstrated that the actuator rate limit nonlinearity can induce severe closedloop instability in a flutter suppression system. An example can be found in Figures 1 and 2. When a designed control law is specified, the actuator design parameters can be tuned (increased actuator rate limit and bandwidth) such that actuator dynamics do not couple with the structural dynamics. However, the controller bifurcation diagrams, shown in Figure 3, revealed that even with proper tuning of the actuator parameters a region of hysteresis was always present; this region may preclude flight control system designers from using full adaptive control and may also result in the requirement for a larger and heavier actuator due to increased rate limits and bandwidth.

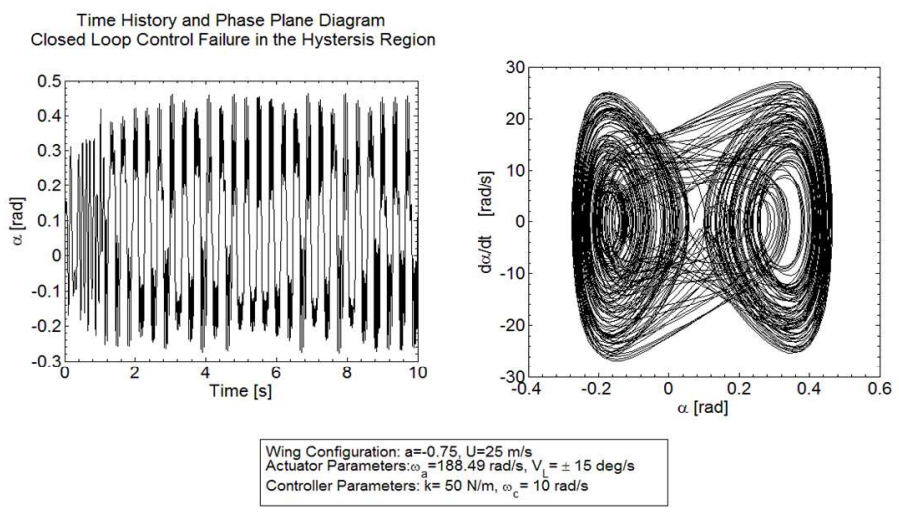

Figure 1 Closed Loop Response in the hysteresis region [4]
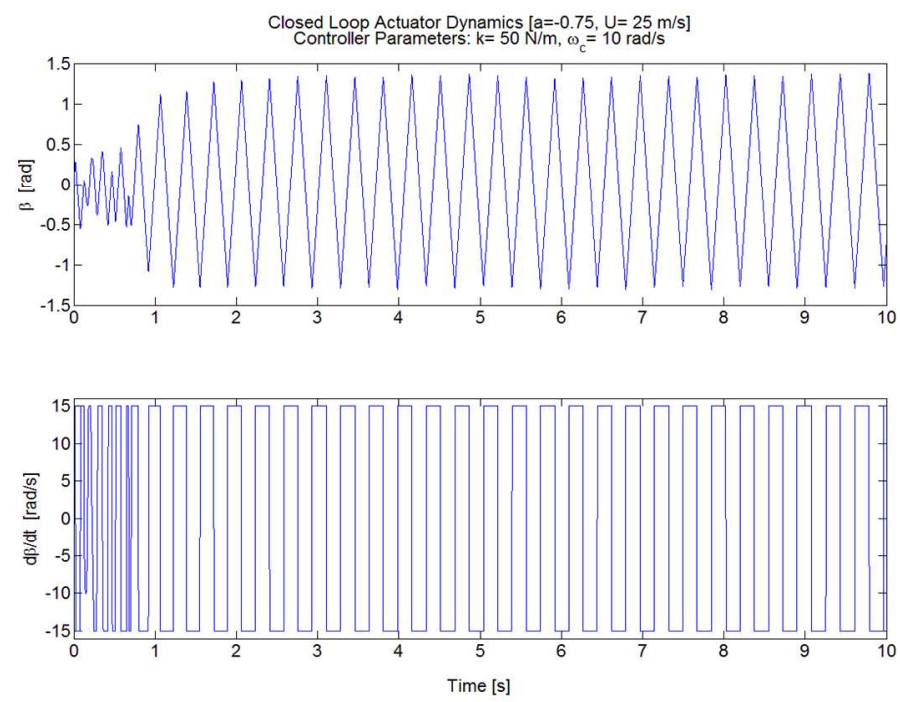

Figure 2 Closed Loop actuator dynamics [4] 


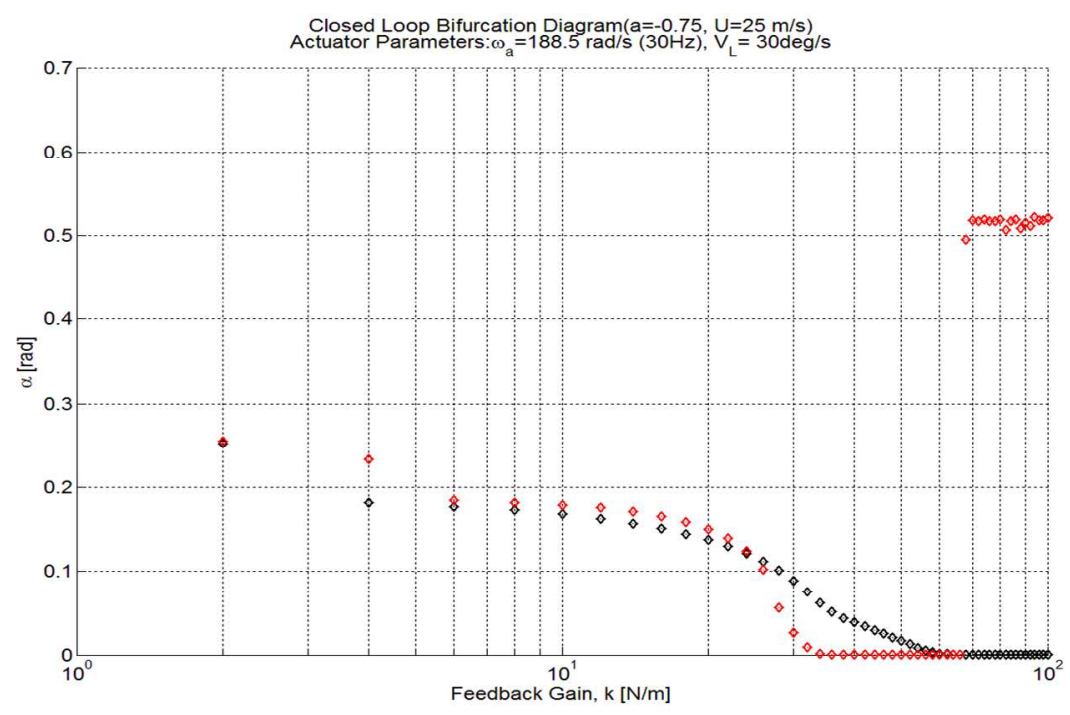

Figure 3 Controller Bifurcation Diagram [4]

In this study, it is the objective to examine whether these nonlinear pre-filters previously examined by a joint Air Force Institute of Technology /Test Pilot School (AFIT/TPS) research program into controlling pilot induced oscillation [6] can be applied to the LCO control problem in aircraft structures. Specifically, the research objectives are to devise the appropriate controlloop configurations for the implementation of the nonlinear pre-filters; for a given elastic axis location, design the filter and actuator parameters such that full suppression of LCOs is achieved; and compare the performance of the filters and make recommendations for further research with respect to NASA's Aviation Safety Program.

The rest of the paper is organized as follows: in Section 2 the nonlinear filter theory is presented, followed system modeling in Section 3. Section 4 discusses the design and implementation of the control architecture followed by conclusions in Section5.

\section{NONLINEAR FILTER THEORY}

The Feedback-with-Bypass (FWB) filter (Figure 4) was designed in 1996 by Dr. Lars Rundqwist for the SAAB JAS -39 Gripen aircraft as a preventative solution to the PIO problem [5] and later employed on the NF-16D VISTA [6]. A command composed of both high and low frequency components enters a low pass filter. High frequency components greater than the cut-off frequency of the first low pass filter will bypass the majority of the filter. The low frequency components will pass through the first SoftWare Rate Limiter (SWRL) which is set to the same value as the actuator rate limit. During rate limiting, the input signal to the SWRL is greater than the output. When this occurs, the difference between the output and input are passed to the second low pass filter. This difference signal has a negative sign; consequently its phase is shifted 180 degrees from the input command. When this signal passes thought the low pass filter and is feed back to the low frequency input, a phase lead is added to the system. The result is a rate-limited signal with substantial less phase lag. 


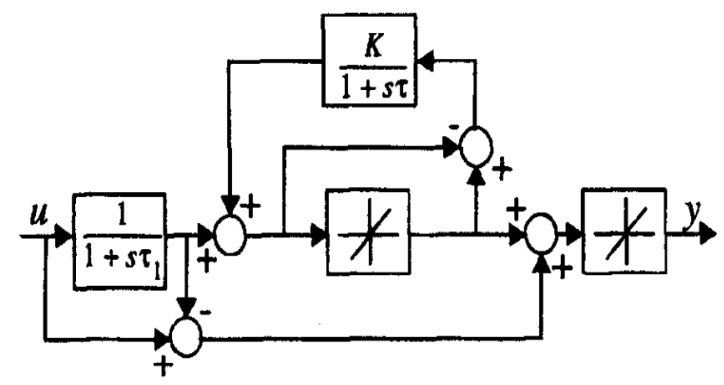

Figure 4 Feedback-with-Bypass Filter [5]

Previous ground and flight test results with the FWB filter demonstrated good performance for different actuator rate limits [6]. The Derivative-Switching (DS) filter or Rate Limit Pre-Filter (RLPF) has three branches, see Figure 5. The upper branch uses an algorithm that differentiates limits and integrates in order to keep the output in phase with the low frequency input as indicated in Figure 6. A reset integrator is used to correct the bias inherent in an unsymmetrical input. The middle branch provides the switching logic. First, high frequency noise is removed from the signal. The rate and acceleration of the filtered signal are compared to preset values. If either derivative exceeds their respective thresholds, then the upper branch is activated. Otherwise the lower branch is active and the signal passes thought the filter without a switching event. A later version of the RLPF contains a second order filter in the third branch to suppress high frequency noise.

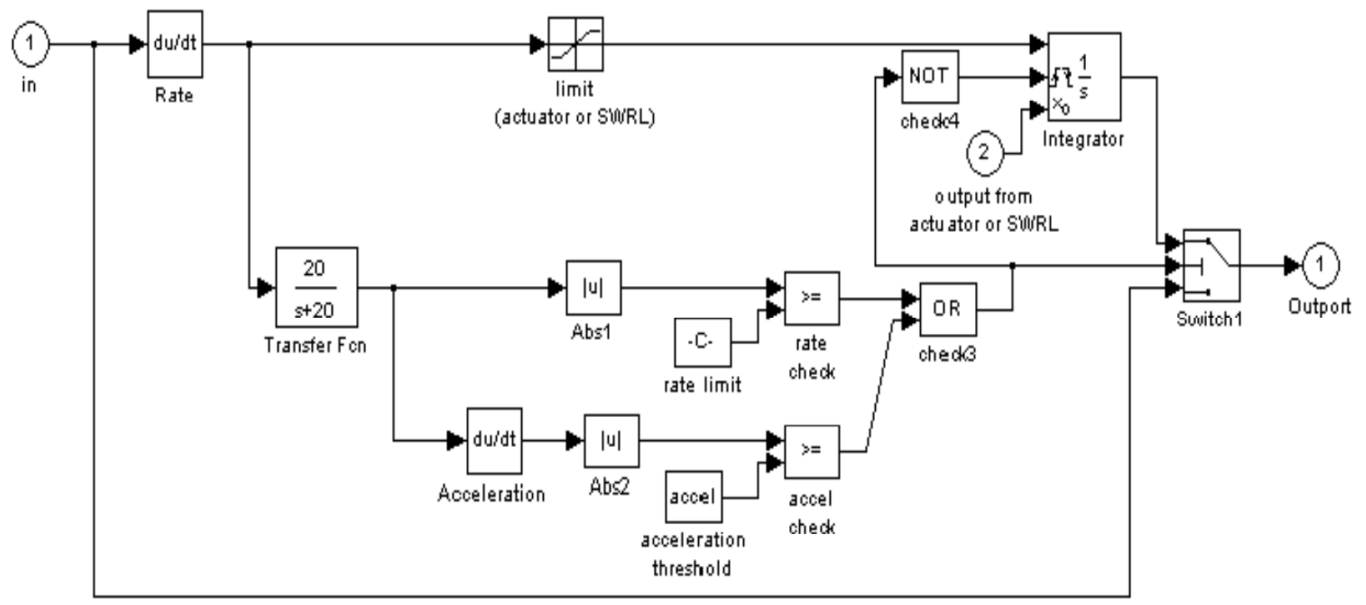

Figure 5 Derivative Switching Filter [6]

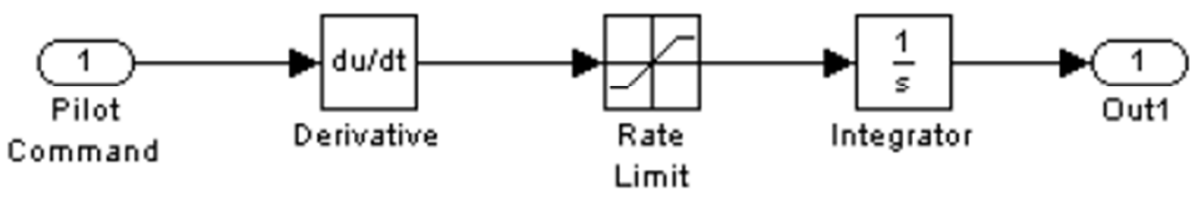

Figure 6 Upper Branch 


\section{NONLINEAR ASE SYSTEM MODELING}

Due to the complexity of the nonlinear actuator models, for this analysis a nonlinear first order rate limited actuator model will be used. This model still retains most of the characteristics of interest including rate limited operations and actuator nonlinearities. The selected model is realistic and it was used successfully in the study of Pilot Induced Oscillations (PIO) [6] in the simulation and flight test of the NF-16D Variable Stability In-flight Simulator Test Aircraft (VISTA). Often the same flight control system actuators allocated to the automatic flight control system (AFCS) are also employed in flutter suppression and hence the same actuator nonlinearities that cause flying qualities issues also cause ASE interactions. However the inherent complexity of an actuator model with multiple nonlinearities made it difficult to isolate the cause of the closed-loop instability. As such the nonlinear first-order rate limited actuator model provides a means for simple parametric investigation and demonstration of the research problem at hand. Common actuator nonlinearities include saturation, friction, dead zones (or free play) and hysteresis and rate saturations. For the purposes of this research, the hard stops on this simulated actuator are set at $\pm 40^{\circ}$.

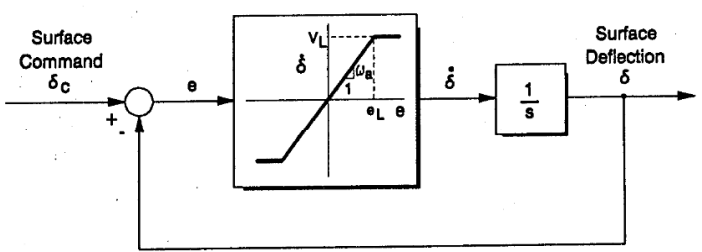

Figure 7 Rate Limited Actuator [8]

The Aeroelasticity Group at Texas A\&M University has conducted a number of experiments using the Nonlinear Aeroelastic Test Apparatus (NATA). The experiments performed have provided the validation for the theoretical model that will be used in this research. The NATA test bed has been used to study both linear and nonlinear aeroelastic behaviour as well as the development of control laws for flutter suppression. The system consists of a NACA 0012 and is controlled by a full span trailing edge control surface located at $20 \%$ chord. The pitch and plunge stiffnesses of the NATA are provided by springs attached to cams with profiles designed to induce specific responses. For example, a parabolic pitch cam yields a spring hardening response. This is the mechanism that causes the NATA to exhibit the limit cycle behaviour. The model parameters are provided by Ko et al. [7], while a MATLAB Simulink ${ }^{\circledR}$ model of this system was supplied in Alstrom [4].

\section{DESIGN AND IMPLEMENTATION}

The FWB pre-filter accepts only a command signal. Initial simulation using the designed LQR control signal showed that the FWB pre-filter transformed the desired control signal into a step input. The implication is this result is that this approach would lead to a non-zero final position of the trailing edge flap even at zero deflection. This could be quite problematic when attempting to assess the effect of the flutter suppression system on the flight dynamics. The Derivative Switching pre-filter, although it is designed to accept both a command or desired control signal and the actuator output, it caused closed-loop instability likely due to the nature of switching 
conditions. Initial results with both these filters require further investigation. One further nonlinear pre-filter design was proposed by Alcala et al. [9]. Specifically, the proposed nonlinear filter consists of a phase lead transfer function, $G_{p}(s)$ and the nonlinear element called a rate limiter. The given structure achieves phase compensation by feeding back the output signal and obtaining the error signal. The error signal is then fed to the phase lead transfer function. The output is summed with the rate limited output. Previous results with coupled rate limited actuator and linear airframe dynamics showed good phase compensation.

The nonlinear filter was modified to accommodate the rate limited actuator (see Figure 7) instead of the rate limiter for this project. The phase lead transfer function is of the form:

$$
G_{p}(s)=K_{p} \frac{s^{2}+\left(4 \pi \zeta f_{1}\right) s+4 \pi^{2} f_{1}^{2}}{s^{2}+\left(4 \pi \zeta f_{2}\right) s+4 \pi^{2} f_{2}^{2}}
$$

This transfer function allows for the specification of the frequency range over which the phase compensation is required. The next step in the design process is to design the feedback gain matrix, K via optimal control or Linear Quadratic Regulator (LQR) theory. For this numerical experiment the elastic axis location of the aeroelastic system is located at the mean aerodynamic center $(a=-0.75)$ and the velocity is $15 \mathrm{~m} / \mathrm{s}$; the resulting aeroelastic mode is $3.6 \mathrm{~Hz}$ [4]. The positive semi definite matrix $\mathrm{Q}$ and the real symmetric matrix $\mathrm{R}$ are given as follows:

$$
\begin{gathered}
Q=\operatorname{diag}\left[Q_{h} Q_{\alpha} Q_{\dot{h}} Q_{\dot{\alpha}}\right] \Rightarrow \operatorname{diag}\left[\begin{array}{llll}
10 & 20 & 10 & 10
\end{array}\right] \\
R=[10]
\end{gathered}
$$

The final closed-loop configuration is shown below in Figure 8. Specifically, the outer loop is formed by the full state feedback signal and the inner loop is formed by flap displacement. Both of these signals form an error signal that is feed-forward the phase lead transfer function. Initial simulation runs with the configuration shown below were conducted with the feedback loops separately. Once the feedback gain matrix, $\mathrm{K}$ has been specified, the actuator dynamics are adjusted such that there is sufficient frequency separation between $3.6 \mathrm{~Hz}$ and the actuator natural frequency i.e. the actuator natural frequency must be greater than $3.6 \mathrm{~Hz}$. For this simulation the actuator frequency is set at $50 \mathrm{~Hz}$. The closed loop system is then subjected to a sharp edged gust. When the outer loop is subjected to the short time sharp edged of 5 sec of time, it becomes clear that even with optimal control, the rate limiting still renders the aeroelastic system closed-loop unstable (Figure 9). An examination of the actuator dynamics reveals that the peaks of normalized commanded flap angle and the flap displacement are out of phase by $0.03 \mathrm{~s}$ (Figure 10). 


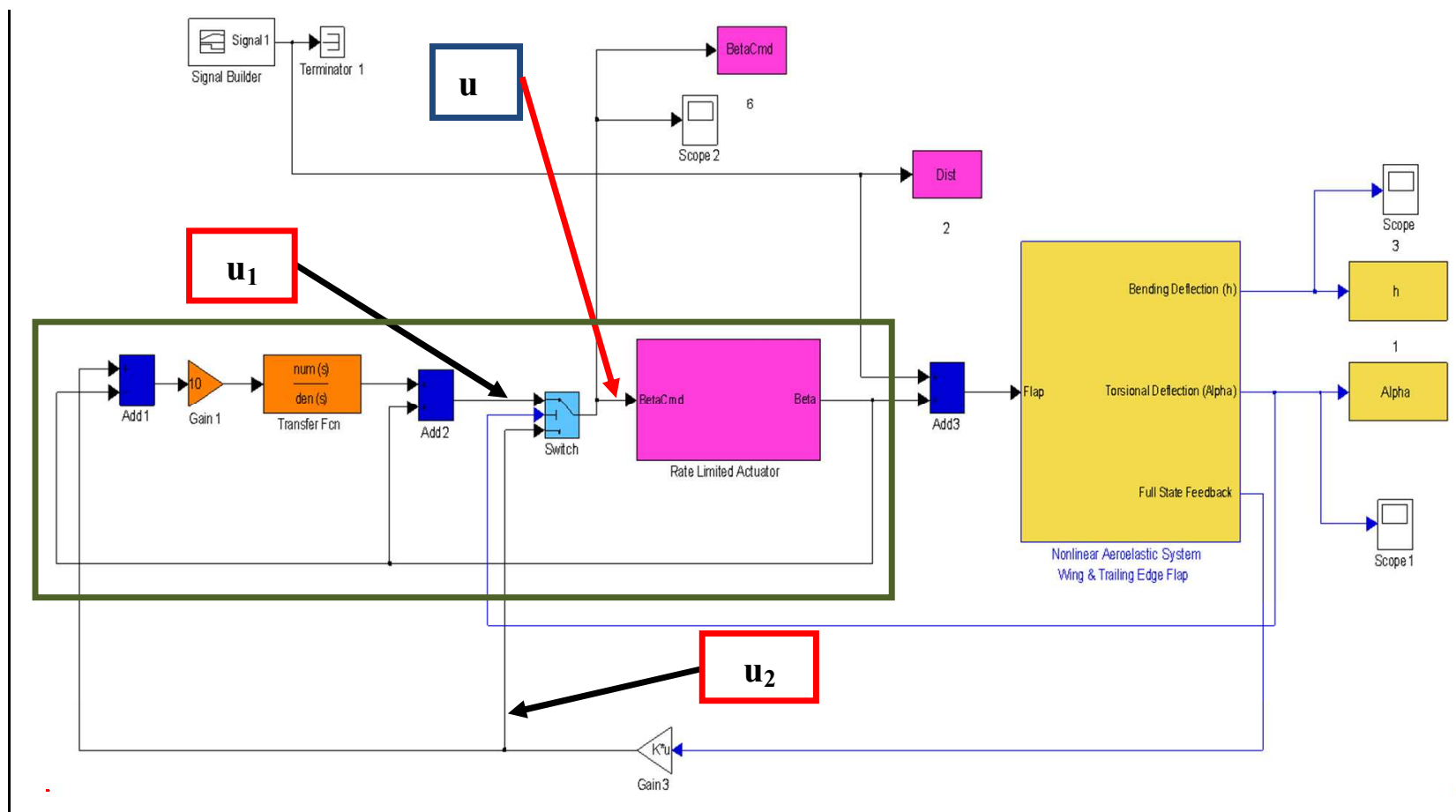

Figure 8 final closed loop configuration (phase compensation in green box)
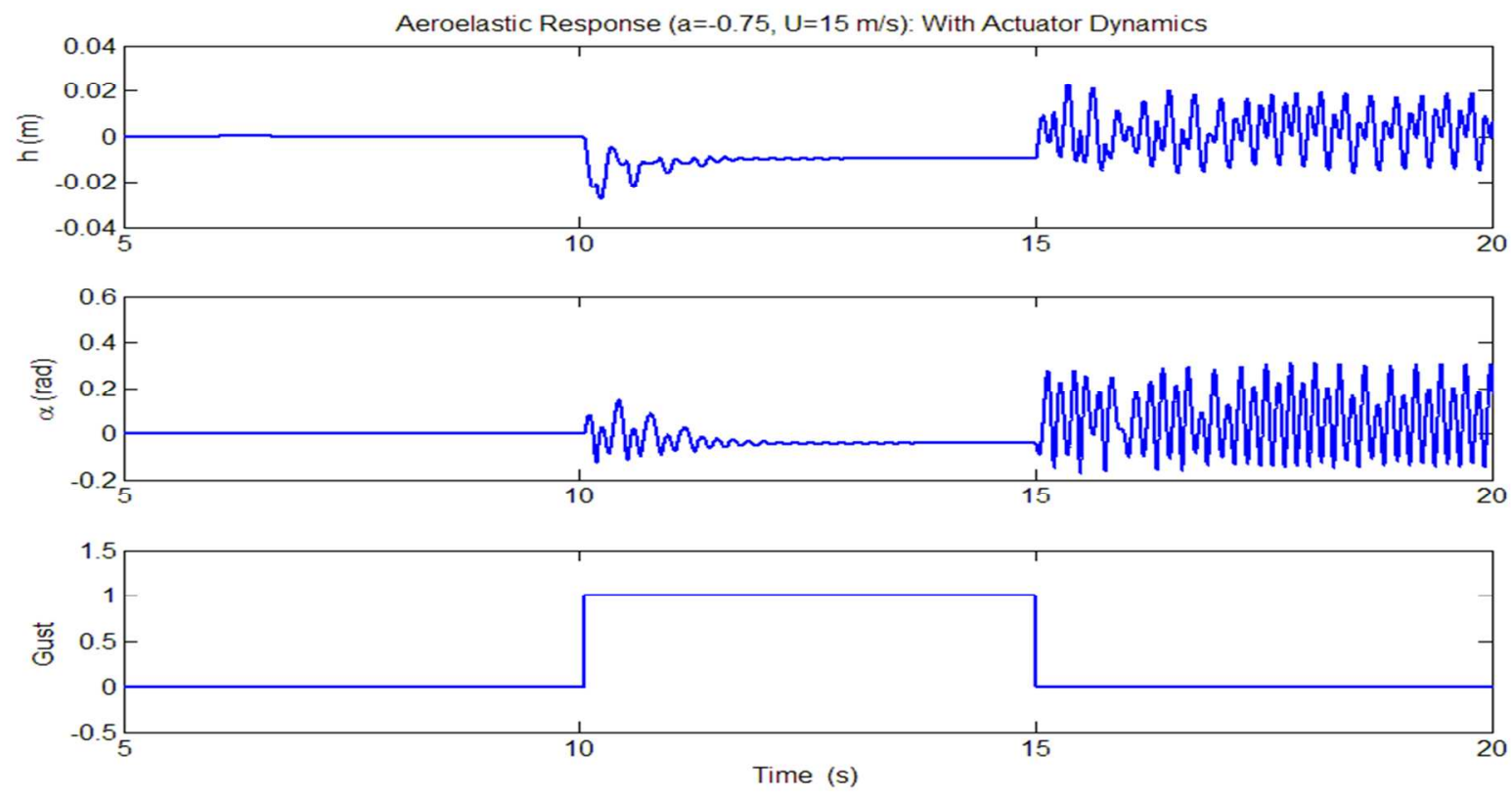

Figure 9 Aeroelastic Response to a $5 \mathrm{sec}$ sharp edged gust 


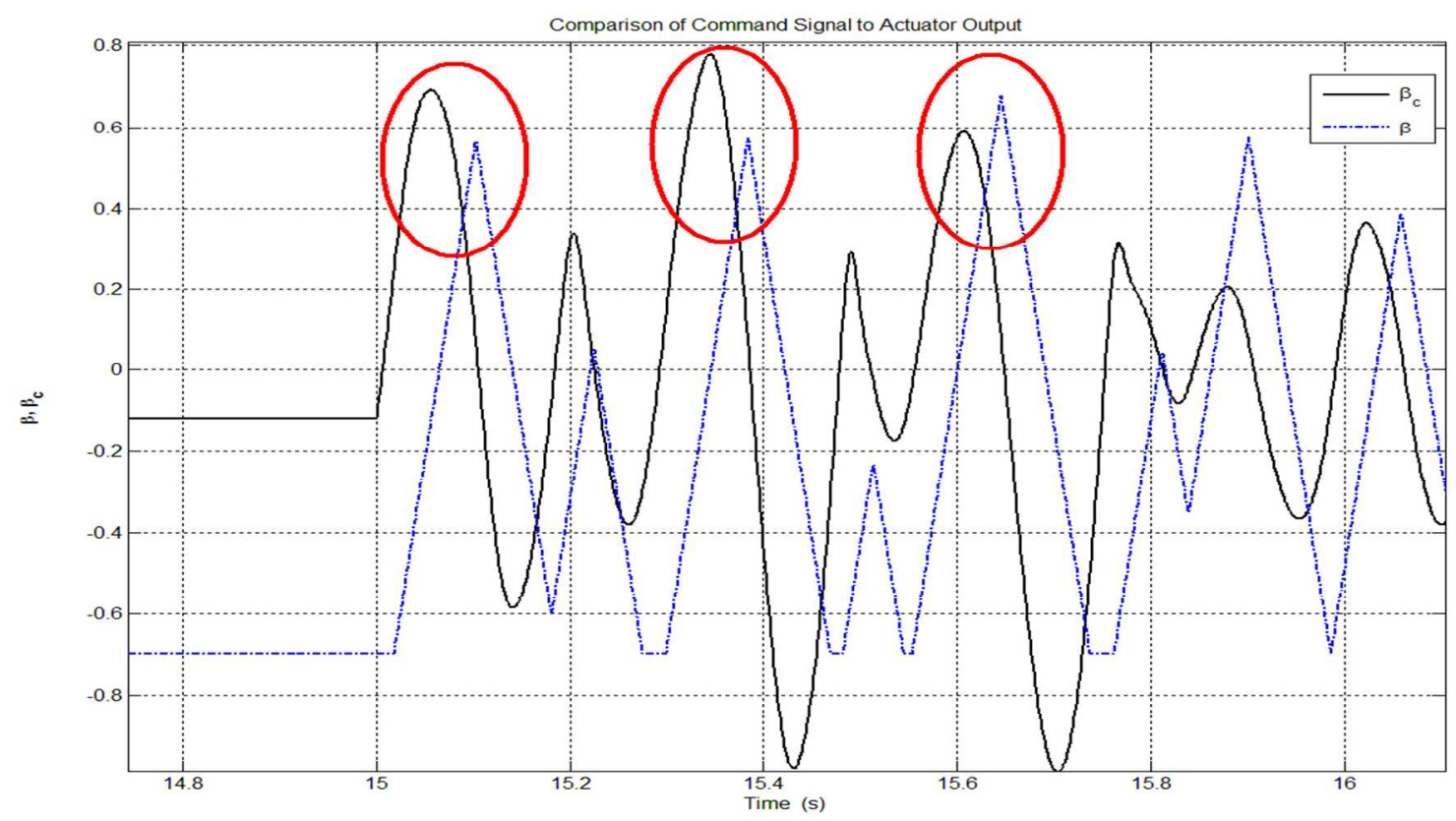

Figure 10 Comparison of Actuator Command with output (outer loop control only)

While adjusting the parameters of the phase compensation loop, mainly the notch filter gain, $K_{p}$, damping ratio, $\zeta$ and the frequency range, $\Delta f=f_{2}-f_{1}$, it was found that when using the phase compensation loop only, a large gain caused a significant steady state offset. That is the final flap displacement after the disturbance has subsided, did not return to a zero position which caused the final position of the airfoil pitch downward. In essence, there are two control signals, one that provides regulation, i.e. it drives the state to zero, and the other provides phase compensation. Therefore in order to take advantage of these controller properties, a simple switching rule must be used. But first, the equations of the feedback control loops must be derived.

Let the error signal $e=u_{L Q R}-\beta$ and $u_{1}=K_{p} G_{p}(s) e+\beta$. Let $u_{2}=u_{L Q R}$. After some algebra, $u_{1}$ and $u_{2}$ can be written in matrix form (see Figure 8 ):

$$
\left[\begin{array}{l}
u_{1} \\
u_{2}
\end{array}\right]=\left[\begin{array}{cc}
K_{p} G_{p}(s) & 1-K_{p} G_{p}(s) \\
1 & 0
\end{array}\right]\left[\begin{array}{c}
u_{L Q R} \\
\beta
\end{array}\right]
$$

The control law is of the following form:

$$
u= \begin{cases}u_{1}, & \alpha>\alpha_{\text {cond }} \\ u_{2}, & \alpha<\alpha_{\text {cond }}\end{cases}
$$

By adding switching condition we have formed a nonlinear controller. This is called a piecewise impulse controller. The control signals $u_{1}$ and $u_{2}$ are hyperplanes for which the control solution will remain on a given plane until the switching condition is met and then it will jump to the other plane. The control solution will continue to do so until the system damping increases to the 
point where the system reaches equilibrium. If one takes the limit of $G_{p}(s)$, we are left with a constant, mainly $K_{p}\left(f_{1}^{2} / f_{2}^{2}\right)$. Substituting this constant into Eq. (3) and setting $K_{p}$ equal to unity, the determinant is found and by setting it equal to zero, the frequency ratio is found to be 1 . So in order for the closed loop system to remain stable, the frequency ratio must be less than 1 . This physically translates to $\Delta f$ not being equal to zero. The ability to invert the matrix in Eq. (3) means that the closed loop system will remain stable in presence of a disturbance. Furthermore Eq. (3) gives us some insight on the adjustment of the controller parameters. Earlier, it was stated that large values of $K_{p}$ caused an offset in the flap angle and wing displacements, however the switching removes the offset even in the presence of a high filter gain (see Figure 11). The reason for this effect has to do with the fact that when the angle-of-attack falls below the switching condition, the optimal control loop then drives the angle-of-attack to zero. Large filter gains, now just cause noisy switching events. Some judgement must be applied when tuning $K_{p}$. Figure 12 shows the switching events. They are best observed by looking at the actuator rate time history. The reader will notice the two sharp edged trajectories and on the third switching event the angle-of-attack fall below 0.05 radians and settles to zero. Next the phase lead network damping ratio was varied from 0 to 1 , with $K_{p}$ set equal to 1 . A step input was used to complete this parametric evaluation. Figure 13 shows the damping ratio plotted against the settling time of the angle-of-attack.

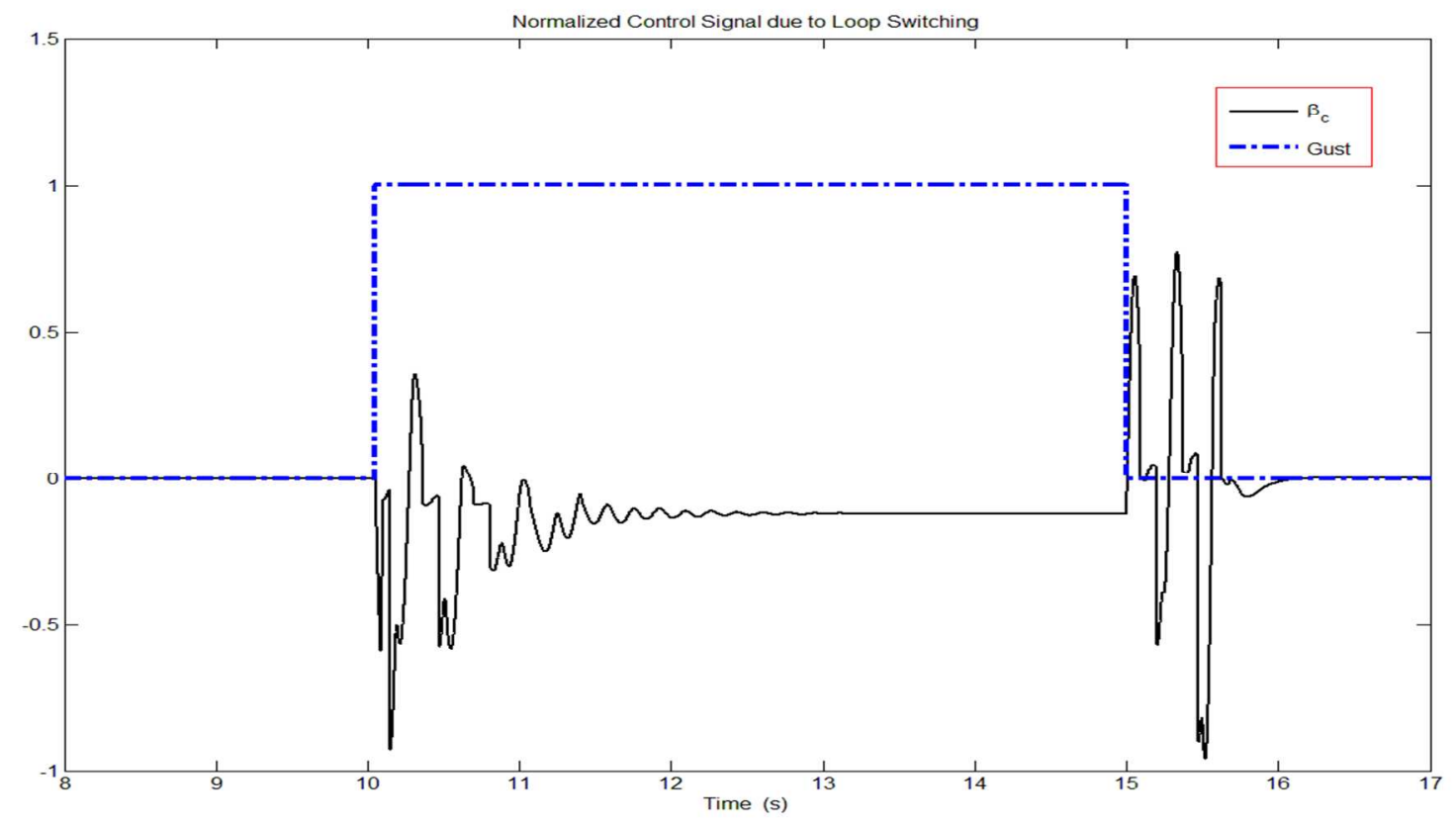

Figure 11 Overlay of $5 \mathrm{sec}$ sharp edged gust with commanded flap angle 
From a damping ratio of 0.08 to 0.2 , note the significant increase in settling time yet for ratios lest than 0.08 , the settling time is $5 \mathrm{sec}$. A similar trend is observed from damping ratios greater than 0.6. It is possible that the range of low damping ratios that are causing the longer settling times are associated with a region of instability. Typically when time delays are involved there are many solutions to the stability equation, but current system is highly nonlinear and it difficult to find these stability region analytically; so hence it must be done numerically. Further investigation is required to adequately quantify the above given assumption and results will be presented elsewhere.

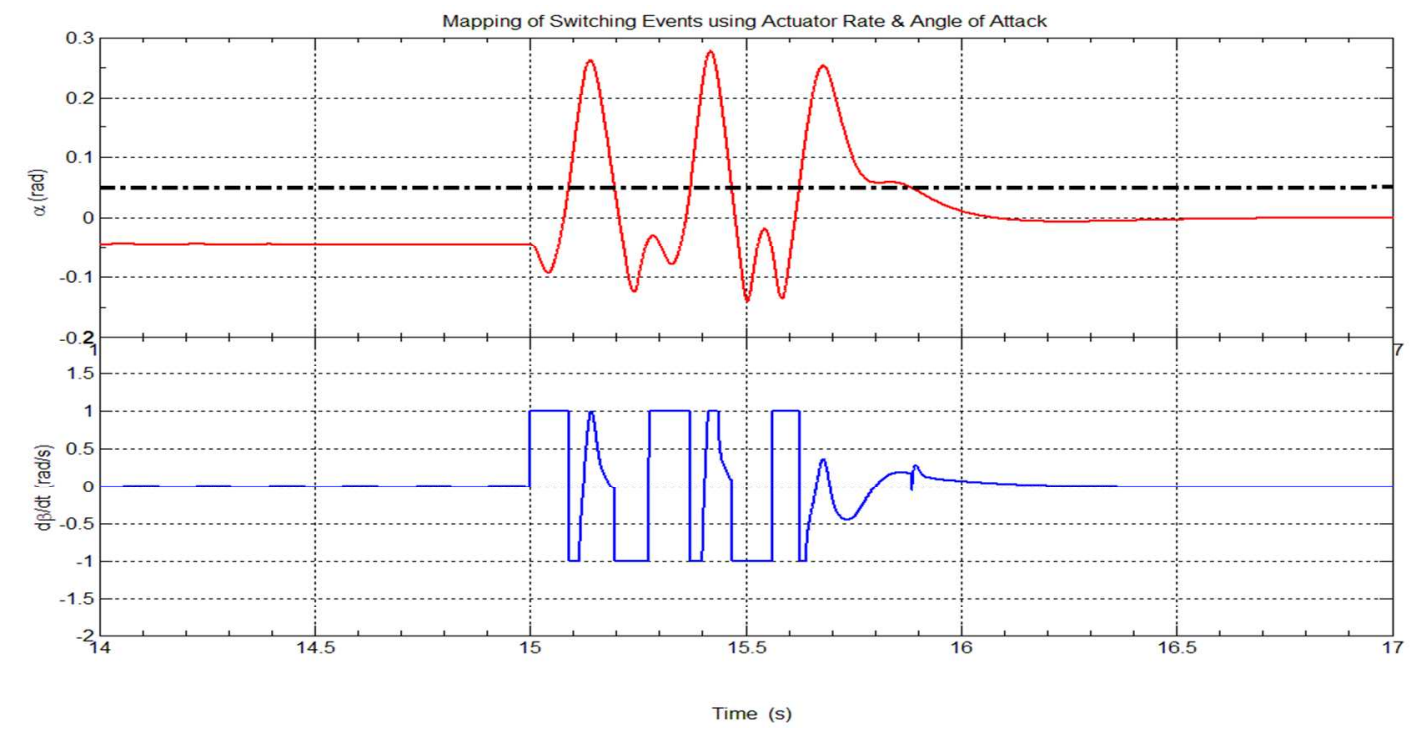

Figure 12 Mapping of switching events

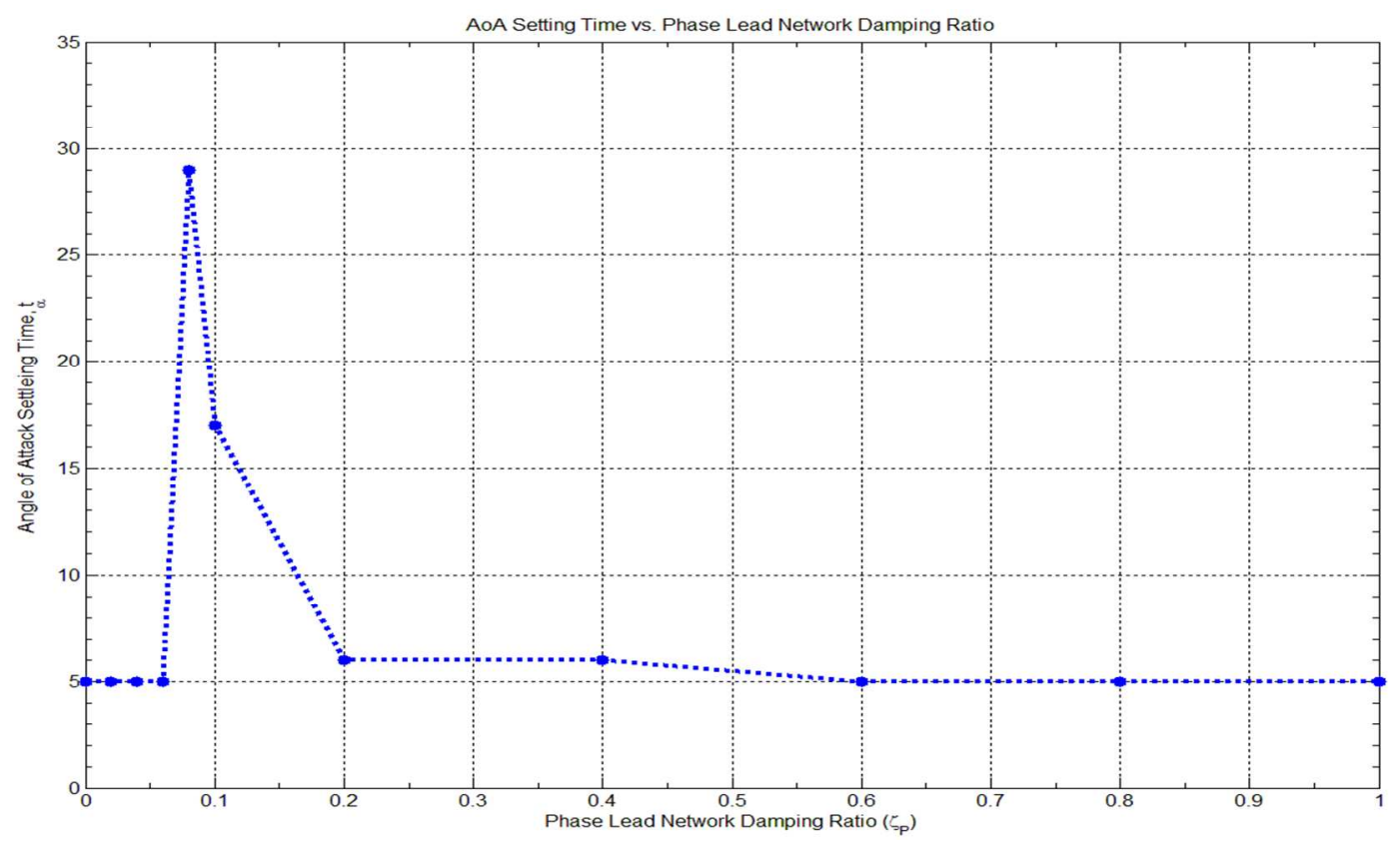

Figure 13 Settling Time vs. Filter Damping Ratio 
The final parameter configuration that have been selected are as follows: For the elastic axis location, $\mathrm{a}=-0.75$ at $\mathrm{U}=15 \mathrm{~m} / \mathrm{s}$, the actuator natural frequency is $50 \mathrm{~Hz}$ with the rate limits, $V_{L}= \pm 15 \mathrm{deg} / \mathrm{s}$, and the filter damping ratio, $\zeta$ is equal to 1 and the frequency range is from $1 \mathrm{~Hz}$ to $20 \mathrm{~Hz}$ with a maximum phase angle of 129 degrees. Figure 14 shows the aeroelastic response when subjected to the $5 \mathrm{sec}$ sharp edged gust and under closed-loop control. Under these modeling assumptions the controller is capable of tracking the disturbance and returning the system to a zero deflection condition. Figure 15 highlights the fact that the controller removes the time delay of $0.03 \mathrm{~s}$ and regulates the angle-of-attack. Note, that inside the green box, it can be observed that the commanded flap angle and the flap displacement are in phase i.e. the time delay is zero.
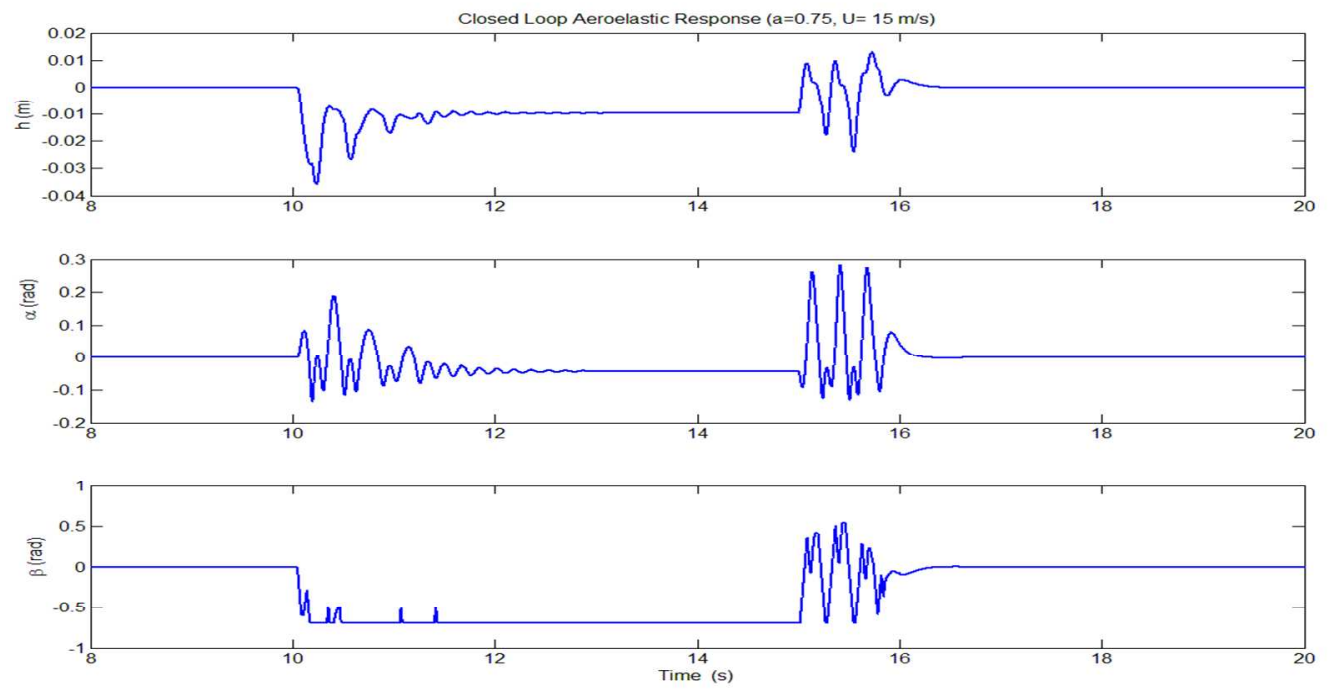

Figure 14 Closed loop response to disturbance

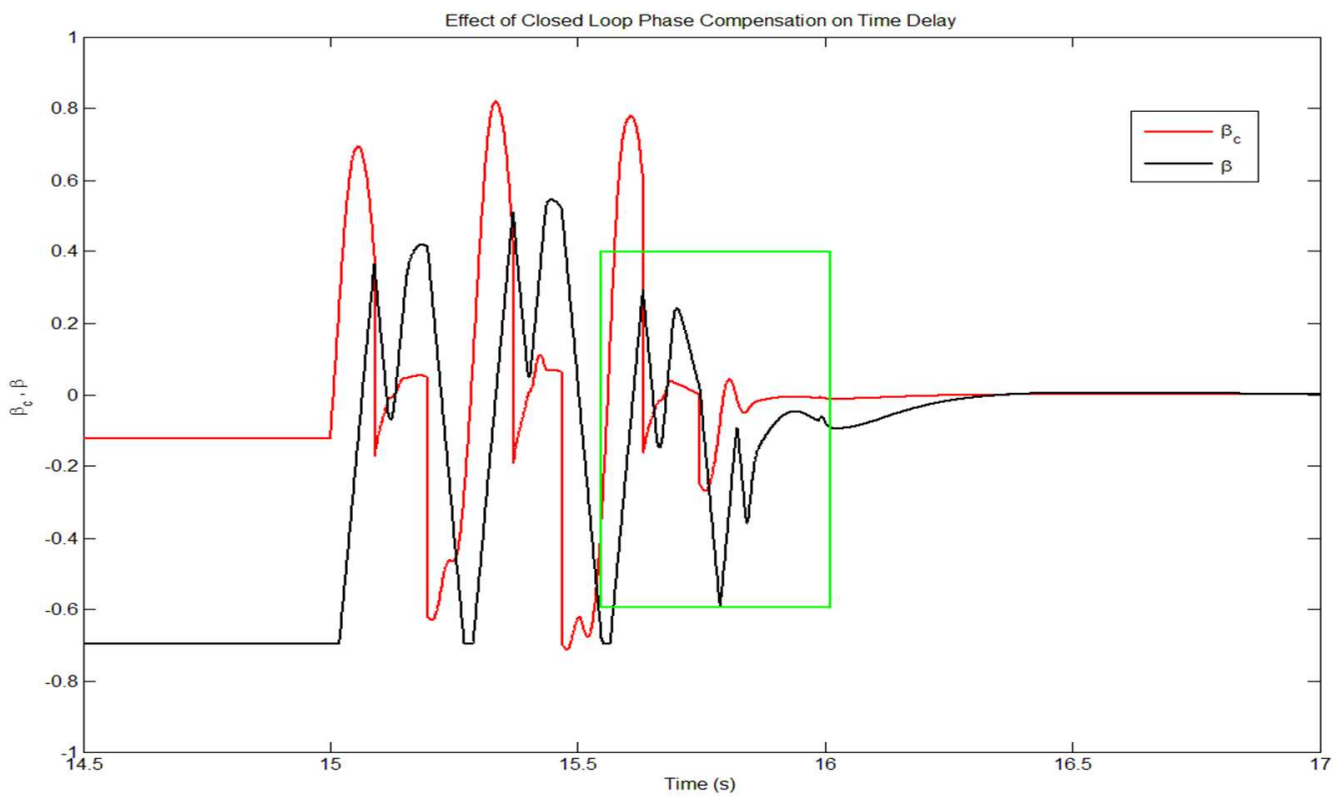

Figure 15 Effect of Controller of time delay 


\section{CONCLUSIONS AND FURTHER OUTLOOK}

In this work the integration of the LQR control with phase compensation, combined with a simple switching rule has been successfully implemented.Although this controller is able to suppress aeroservoelastic coupling, when subjected to a short-time sharp edged gust, it not capable of addressing a frequency based disturbance. This is a limitation that must be considered by the designer. With respect to the pre filters initially proposed, a review of the literature on nonlinear pre-filters shows that the plant dynamics in all simulation were linear. It is possible that using surrogate rate limiters (FBW) and actuator dynamics (Derivative Switching filter) was adequate for the aircraft handling qualities evaluation, But for highly nonlinear simulations such as the one presented in this paper, it is likely more effective to wrap the phase compensation architecture around the actual actuator dynamics itself. This approach may work well when merging the flight software with the hardware; that is the phase compensation can be a flight software implementation which requires the actuator signals in order to close the loop. A follow on study to this might be to evaluate the phase compensation properties of adaptive noise control (ANC) techniques for aeroservoelasticity; should the ANC techniques prove not to have such properties then a hybrid controller will have to be explored rigorously, i.e. a phase compensation loop should be considered in conjunction with a disturbance rejection loop to make progress toward the implementation of flight worthy adaptive control algorithms for aeroservoelasticity.

\section{REFERENCES}

1. NASA Integrated Resilient Aircraft Control, May 2009, http://www.aeronautics.nasa.gov/nra_pdf/irac tech_plan_cl.pdf

2. Li, Xiaohong, L., Brenner, Martin J., "Practical Aeroservoelasticity In-Flight Identification and Adaptive Control", AIAA GNC/AFM/MST/ASE 2010 Toronto Ontario, Canada.

3. Department of Defense, Flying Qualities of Piloted Aircraft, MIL-STD-1797, 19 December, 1997.

4. B. Alstrom, P. Marzocca, E. Bollt, G. Ahmadi, "The Effect of Rate Limited Actuator Dynamics on the Adaptive Control of a Nonlinear Aeroservoelastic (ASE) System," AIAA-2011-1769, 4-7, 2011, Denver, CO.

5. Rundqwist, Lars, Stahl-Gunnarsson, Karin, "Phase Compensation of Rate Limiters in Unstable Aircraft", Proceedings of the IEEE International Conference of Control Applications, Dearborne MI, Sept. 15-18, 1996.

6. Hanley, James G., "A Comparison of Nonlinear Algorithms to Prevent Pilot Induced Oscillations caused by Actuator Rate Limiting”, Masters Thesis, AFIT/GAE/ENY/03-4, 2003, Wright Patterson AFB, Dayton OH.

7. Ko et al., "Nonlinear Control of a Prototypical Wing Section with a Torsional Nonlinearity", Journal of Guidance, Control and Dynamics, Vol. 20, No. 6, Nov-Dec 1997.

8. Kylde, David H, Unified Pilot-Induced Oscillation Theory, Volume 1:PIO Analysis with Linear and Nonlinear Effective Vehicle Characteristics, Including Rate Limiting.WL-TR-96-3028. AFRL, Wright Patterson AFB OH, December 1995. 
9. Alcala, I, Gordillo, F. and Aracil, J. 'Phase compensation design for prevention of PIO due to actuator rate saturation', 2004 American Control Conference, Boston, MA June 30-July 2, 2004 